\title{
Soft selection and quantitative genetic variation: a laboratory experiment
}

\author{
AURORA GARCÍA-DORADO, PALOMA MARTIN \& NIEVES GARCÍA \\ Departamento de Genética, Facultad de Biologia, Universidad Complutense, 28040 Madrid, Spain
}

\begin{abstract}
The effect of environmental heterogeneity on the genetic variation of different quantitative characters was studied in two laboratory and two recently captured populations of Drosophila melanogaster. Two different culture media (habitats $\mathrm{R}$ and $\mathrm{G}$ ) were used. Coarse-grained heterogeneity with independent density control in each habitat $(R+G)$, and fine-grained $(R / G)$ heterogeneity were simulated in population cages. Control populations in both $R$ and $G$ habitats were also maintained.

Genetic differences for oviposition-site preference, larval preference and/or within-habitat viability were found between subpopulations sampled from different media. This happened in all four populations maintained on $R+G$, two populations maintained on $R / G$, and one control population. Thus, environmental heterogeneity seems to protect genetic variability responsible for between-habitat genetic differentiation, particularly when such heterogeneity corresponds to the 'soft selection' model $(\mathrm{R}+\mathrm{G})$. However, for the quasi-neutral trait sternopleural bristle number, no genetic between-habitat differentiation, nor increased heritability were observed in populations maintained under any kind of environmental heterogeneity. Hence, although soft selection seems to be a real force in determining adaptation to heterogeneous environments, the genetic variability maintained may be small in relation to the whole genome.
\end{abstract}

Keywords: environmental heterogeneity, selection, soft selection.

\section{Introduction}

Environmental heterogeneity has often been invoked as a factor in the maintenance of genetic variability. Some Drosophila laboratory experiments support this view (McDonald \& Ayala, 1974; Powell \& Wistrand, 1978; all using recently captured populations) while others question its general relevance (Haley \& Birley, 1983; Minawa \& Birley; 1978; Yamazaki et al., 1983; all using laboratory populations). Even in those cases where environmental heterogeneity is associated with increased genetic variability, there is no consensus on the mechanism responsible, and the view that heterozygosity 'per se' confers on the individual a greater adaptation to heterogeneous environments has little empirical support (Lerner, 1954; MacKay, 1981). However, under high density, no heterozygous advantage is required to protect a polymorphism in a heterogeneous habitat (Maynard Smith \& Hoekstra, 1980): soft selection (Levene, 1953; Prout, 1968), where the contribution of different habitats is constant over time, may protect non-overdominant polymorphisms when different genotypes are in advantage in different habi- tats. This requires independent density control in each habitat, and that each individual undergoes natural selection within a single habitat (i.e. coarse-grained heterogeneity). Polymorphism protection occurs only under a narrow range of circumstances defined by the contribution of the different habitats and by the relative within-habitat genotypic advantages, so that the model is not very robust (Maynard Smith \& Hoekstra, 1980; Hoekstra et al., 1985). Genetic variability for habitat preference increases its robustness. For this to occur, the environmental heterogeneity must be fine-grained during habitat choice (so that each individual experiences different habitats before choosing one), but coarse grained when natural selection is acting (every individual undergoes selection in the chosen habitat). Genetic variability with respect to both habitat preference and relative within-habitat adaptation may then evolve (García-Dorado, 1986, 1987, 1990; Rausher, 1984, 1985), increasing within-population heterozygosity and perhaps allowing sympatric speciation (Maynard Smith, 1966; Barton et al., 1988; Guittenberg, 1988). 
Most experimental evidence in this field comes from electrophoretic polymorphisms. However, a small sample of enzymatic loci, chosen by experimental convenience, cannot be expected to be directly related to differential adaptation to the habitats considered. Negative results are not surprising, and positive ones may be due to linkage between the loci studied and others.

Appropriate evidence is better obtained by analysing the relationship between environmental heterogeneity and any genetic differentiation for adaptation to (and preference for) these habitats (e.g. Verdonk, 1987 , with $D$. melanogaster). Genetic variability for habitat preference has been found in various species of Drosophila (Cavener, 1979; Jaenike \& Grimaldi, 1983; Jaenike, 1987; Bird \& Someonoff, 1986; Rice, 1985; Rice \& Salt, 1988) and in other groups (Jones, 1982, with Cepea; Thompson, 1988, with swallowtail butterflies). Such variability is sometimes positively associated with within-habitat adaptation (Abdel-Rehim, 1983; Via, 1986; Jones, 1980; Jones \& Probert, 1980; Taylor \& Condra, 1983; Singer \& Thomas, 1988).

The relevance of soft selection to the maintenance of genetic variability could be assessed by studying the genetic variance of quantitative characters (other than principal fitness components) in populations that occupy homogeneous or heterogeneous environments. Loci that control the genetic variation of such characters are a sample in which heterozygosity may be related to environmental heterogeneity because of the role of the character in adaptation to different habitats. MacKay (1981), using D. melanogaster, reported greater genetic variability for sternopleural bristle number (SBN) and for body weight in heterogeneous laboratory environments. As there was no genotype-habitat interaction for these characters, she concluded that the mechanism responsible was heterozygous advantage in a heterogeneous environment. Nevertheless, genotype-habitat interaction for fitness (resulting in no genotype-habitat interaction for these characters) may maintain (via soft selection) genetic variability for a character: for example, if natural selection favours different body weights at different habitats (García-Dorado, 1990), or if a fixed body weight was selected everywhere but habitats differ in their environmental effect on the character's expression. Nevertheless, results concerning SBN are appealing because this character seems to be under little selection in the homogeneous standard laboratory medium (Robertson, 1967).

Here we investigate the genetic mechanisms of adaptation to heterogeneous environments, the prevalence of soft selection, the possible evolution of differentiation for habitat preference under soft selection, and the effect of these phenomenon on the genetic variability of SBN. Two laboratory and two recently captured populations were studied and two different media ( $R$ and $G$ ) were used. Two different heterogeneous environments were simulated. In one of them $(\mathrm{R}+\mathrm{G})$, half the bottles in the population cages contained medium $R$ and the other medium $G$. In the other $(\mathrm{R} / \mathrm{G})$, all bottles in a cage contained adjacent hemicylinders of each medium. For each base population, two controls (one maintained on $\mathrm{R}$ and the other on $\mathrm{G}$ ), and two environmentally heterogeneous populations (one maintained on $R+G$ and the other on $R / G$ ) were kept. As the total number of adults is constrained by the amount of medium, soft selection may occur in the $\mathrm{R}+\mathrm{G}$ heterogeneous environment and variability for oviposition-site preference might evolve. In the $\mathrm{R} / \mathrm{G}$ cages, environmental heterogeneity is fine grained at the larval stage (unless larvae develop strong preference or fidelity), and larvae can move to the less crowded habitat, so that density control is not independent in different habitats. Soft selection is hence not expected to maintain genetic variability, but if heterozygous advantage is associated with the heterogeneous environments, such environment should maintain at least as much variability as the coarse-grained one.

\section{Materials and methods}

Four base populations (A, B, C, D) of D. melanogaster were used. Population $A$ had been captured from a cellar in Ciudad-Real (100 fecundated females) and population B from a vineyard in Valencia (1000 fecundated females), 2 and 7 years before the experiment. Both were maintained on yeast-sugar-sacharose medium until the experiment started. Population $C$ was captured in June, 1985 from the neighbourhood of a cellar in Cordoba ( 60 fecundated females), and population D in September, 1985 from an orchard in Zaragoza (93 fecundated females). The experiment started in January, 1985 for populations A and B, and at one generation after capture for populations $\mathrm{C}$ and D.

The media used are listed below.

Standard: yeast-agar-sacharose medium, not used for population maintenance during the experiment.

$\mathrm{R}$ medium: standard medium stained with McCormick red, containing 15 per cent ethanol by volume, added after the medium had cooled to $85^{\circ} \mathrm{C}$ to prevent evaporation.

G medium: yeast-corn flour-lactose-sacharose medium stained with McCormick blue (which gives a final green colour).

All populations were maintained in $10-1$ plastic cages, each provided with eight plastic $125-\mathrm{ml}$ bottles. 
Each bottle contained $50 \mathrm{ml}$ of medium. Four types of cage (treatments) were used:

Cage R: all bottles containing $\mathrm{R}$ medium.

Cage $\mathrm{G}$ : all bottles containing $\mathrm{G}$ medium.

Cage $\mathrm{R}+\mathrm{G}$ : half the bottles containing $\mathrm{R}$ medium and the other half $\mathrm{G}$.

Cage $\mathrm{R} / \mathrm{G}$, all bottles containing two adjacent $25-\mathrm{ml}$ hemicylinders of each medium ( $R / G$ bottles).

In each cage, all bottles were removed every 21 days and replaced. In the $R+G$ cages, the positions assigned to bottles with different media were alternated every generation.

From each base population, four experimental populations were started, each maintained in a different type of cage. Samples of eggs were obtained from bottles with fresh medium introduced in the cages over $24 \mathrm{~h}$.

\section{Measurement of between-habitat genetic differentia- tion for viability and habitat-preference}

This tested whether individuals occupying the two media ( $R$ and $G$ ) differed in genotype with respect to habitat-preference or within-habitat adaptation.

Measurements were made at two different moments: $\alpha 1$ and $\alpha 2,23$ and 43 months after the experiment started, respectively. The experimental design (Fig. 1) was as follows: (al) All populations maintained under heterogeneous conditions were measured. For R/G populations, egg samples were collected in two $\mathrm{R} / \mathrm{G}$ bottles. Three days later, after the larvae had explored the food, an R (and a $G$ ) bottle was built up by joining the two $R(G)$ hemicylinders from the two R/G bottles. After emergence, 30 pairs (male and female) were sampled from each bottle. We will denote the samples obtained from medium $R$ and $G$ and the strains derived from them as SMR and SMG, respectively. In order to test the existence of between-habitat genetic differentiation, we must use individuals reared in similar habitats. Thus, each sample (SMR and SMG) was transferred to two new bottles with standard medium. From each of these bottles, 30 virgin female and 30 male offspring were sampled and put together in a new bottle with standard medium for 4 days. Sixty pairs were then randomly made for each strain, and each of them was placed in an $\mathrm{R} / \mathrm{G}$ tube $\left(2.3 \mathrm{~cm}^{2} \phi\right)$. Adults were removed after $24 \mathrm{~h}$ and the number of eggs laid on each half of the medium scored. Three days later (after larvae had moved widely through the food) each of the halves were transferred to a new tube with fresh medium of the same type. The number of adults per tube was scored when emergence was completed. This process was performed twice for populations $A(R / G)$ and $B(R /$ $\mathrm{G}$ ) but adult number was not scored the first time.

We became aware of an article by Hoffman (1985),

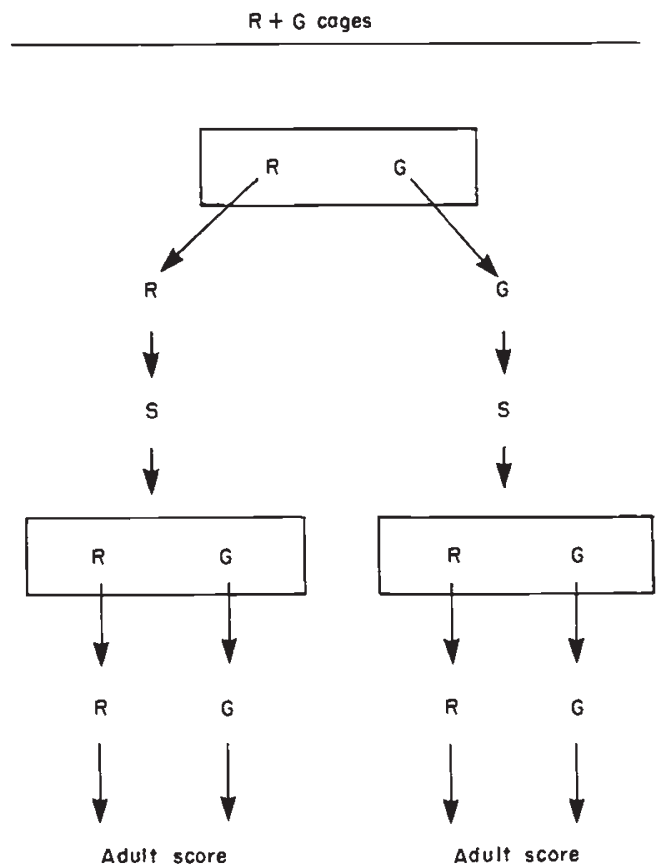

Fig. 1 Scheme for the evaluation of the between-habitat genetic differentiation for viability and habitat-preferences. $\mathrm{t}=$ Generations, $\mathrm{S}=$ standard medium. *In the first evaluation this phase was not performed in cages, but each female layed on a different $R / G$ individual tube. $†$ Only at the first evaluation. 
when these measurements were already in progress, which suggests that the stimuli that determine oviposition-site choice depend on experimental conditions. This induced us to modify the design of the remaining evaluations $(R+G$ cages and second measurement) to assess the preferences in the conditions under which the cages had previously been maintained. For $\mathrm{R}+\mathrm{G}$ populations, after egg sampling, 30 pairs were collected from an R (SMR) and 30 pairs from a $G$ bottle (SMG). They were placed in four 0.25-1 bottles with standard medium (15 pairs to each) and were removed after oviposition. All the flies emerging from the two bottles of the same sample (SMR or SMG) were transferred to an $\mathrm{R}+\mathrm{G}$ population cage containing five bottles of each medium. Thus, we had an $R+G$ cage for the SMR strain, and another $\mathrm{R}+\mathrm{G}$ cage for the SMG strain, both cages having the $\mathrm{R}$ and $G$ bottles in the same positions. The egg number was scored after $24 \mathrm{~h}$ in five specific cells $\left(0.36 \mathrm{~cm}^{2}\right)$ of a net randomly placed on the medium. The total number of adults emerging in each bottle was also scored.

(a2) This evaluation was carried out in all populations, whether maintained in homogeneous or heterogeneous environments. In order to improve the sample's quality, egg samples were obtained from each of four bottles of each medium (in the case of $\mathrm{R} / \mathrm{G}$ populations, these eight bottles were built up from eight R/G bottles, as in section $\alpha 1$ ) following the procedure described above. A set of 15 pairs of adults was sampled from each bottle, transferred to a $0.25-1$ bottle with standard medium, and removed 4 days later. All adult offspring that emerged from the four bottles and that corresponded to the same strain (SMR or SMG) were transferred to a single population cage with either five $R$ and five $\mathrm{G}$ bottles (for treatments $\mathrm{R}+\mathrm{G}, \mathrm{R}$ and $\mathrm{G}$ ) or 10 $R / G$ bottles (for treatment $R / G$ ). There they were allowed to lay for $24 \mathrm{~h}$, and the number of eggs in each bottle was scored in five cells of a net, as in evaluation $\alpha 1$. For populations $R / G$, five $R$ and five $G$ bottles were built up before eggs were scored.

There was a very high density at the larval phase. Under such conditions, the proportional contribution of the habitats is probably not constrained by egg number or by larval viability and preference. Therefore, adult emergence was not scored at $\alpha 2$.

For both $\alpha 1$ and $\alpha 2$, the proportion of eggs $\left(p_{\mathrm{e}}\right)$ laid on $\mathrm{G}$ medium and, when scored, that of emerged adults $\left(p_{\mathrm{a}}\right)$ from the same, was computed for every tube or cell. We tested then, whether these proportions were greater for the SMG than for the SMR sample using one-tailed $t$-tests. Orientative two-tailed $t$-tests were also performed when the sign of the observed differ- ence was opposite to that expected (i.e. when $d_{\mathrm{e}}=p_{\mathrm{e}}(\mathrm{SMG})-p_{\mathrm{e}}(\mathrm{SMR})$ or $d_{\mathrm{a}}=p_{\mathrm{a}}(\mathrm{SMG})-p_{\mathrm{a}}(\mathrm{SMR})$ were negative).

For all measurements performed on population cages (egg scored in cells within bottles), the data had a paired structure because of the position of the cell in the net and that of the bottle into the cage. This was taken into consideration in the statistical analysis [except for the first evaluation of $C(R+G)$, where that paired structure was unrecorded, which may have reduced the power of the corresponding test]. Average proportions over the five cells recorded per bottle were used as an estimate of the bottle's value. Therefore, only 4 degrees of freedom were left for comparisons $[8$ for the first evaluation of $C(R+G)]$.

These measurements were primarily designed to detect between-habitat genetic differentiation for oviposition-site preference. In the first evaluation, the number of adults was also scored in order to obtain an insight into the between-habitat differentiation for both larval preferences and within-habitat adaptation (measured by the survival probability).

In the $\mathrm{R}+\mathrm{G}$ cages (as in the controls), $p_{\mathrm{a}}$ (the proportion of adults emerged from $G$ ) depends on the oviposition-site preference, the larval within-habitat adaptation and the larval density.

In the $\mathrm{R} / \mathrm{G}$ populations, $p_{\mathrm{a}}$ depends on larval density, preferences and within-habitat adaptation. Oviposition-site preference is irrelevant unless larvae exhibit high habitat fidelity, or some kind of behaviour conditioned by (or genetically associated with) the medium where the egg was placed. Nevertheless, as the SMR and the SMG strains were split at the larval stage, differentiation for oviposition-site preference could not be observed unless genetic between-habitat differentiation occurred at the larval phase.

\section{Sternopleural bristle number (SBN) heritability}

The heritability of SBN (total number on both plates) was estimated in a standard medium as the regression of the mean offspring score on the mid-parental value. The design was optimized by practicing divergent selection with assortative mating (Hill, 1970). Estimates were obtained in December, 1986 for all populations. Therefore, 24, 24, 19 and 16 generations had elapsed from the beginning of the experiment for populations A, B, C and D, respectively. In November, $1987, R$ and $G$ populations were obtained from each $\mathrm{D}(\mathrm{R}+\mathrm{G})$ and $\mathrm{D}(\mathrm{R} / \mathrm{G})$. SBN heritabilities for these six populations [four new controls, $D(R+G)$ and $D(R / G)$ ] were estimated in February, 1989 using the same procedure. 


\section{Measurement of between habitat genetic differentia- tion in sternopleural bristle number}

Measurements were obtained for populations that experience environmental heterogeneity $(\mathrm{R}+\mathrm{G}$ and $\mathrm{R} / \mathrm{G}$ ) every 4 months up to a total of four evaluations for populations $\mathrm{A}$ and $\mathrm{B}$ and three evaluations for populations $\mathrm{C}$ and $\mathrm{D}$.

In treatments $R+G$, egg samples were obtained from an $R$ and a $G$ bottle. In treatment $R / G$, egg samples were obtained from two $R / G$ bottles, from which an $\mathrm{R}$ and a $\mathrm{G}$ bottle were built up 3 days later. From the emerging adults, 120 fecundated females and 120 males were sampled from each bottle and paired. Each pair was placed at random in a tube from which offspring were obtained: $60 \mathrm{R}$ tubes and $60 \mathrm{G}$ tubes. Two female offspring were scored for SBN in 50 tubes of each type. Thus, each group of 50 tubes can be classified with respect to the medium from which the parents had been sampled (sampling medium, SM) and the medium in the tube (nursing medium, NM). The data structure is that of a completely balanced design, and a two-way analysis of variance was used corresponding to the model

$x_{i j k l}=\mu+\alpha_{i}+\beta_{j}+\alpha \beta_{i j}+\delta_{k(i j)}+\varepsilon_{l(i j k)}$,

where $\mu$ is the mean, $\alpha$ and $\beta$ are the fixed effects of factors SM and NM, respectively (each with two levels, $\mathrm{R}$ and $\mathrm{G}$, and $\nu=1$ degree of freedom), $\alpha \beta$ is the interaction fixed effect $(v=1), \delta$ is the random effect of the factor 'tubes' $(v=196)$ nested within the interaction, and $\varepsilon$ is the random error to be estimated from the individual measurements within tubes $(v=200)$.

\section{Results}

Differences $\left[d_{\mathrm{e}}=p_{\mathrm{e}}(\mathrm{SMG})-p_{\mathrm{e}}(\mathrm{SMR})\right]$ between the proportions of eggs laid on medium $G$ by $S M G$ and SMR females are shown in Table 1 , as are the corresponding differences between the proportion of adults that emerged from the same medium $\left[d_{\mathrm{a}}=p_{\mathrm{a}}(\mathrm{SMG})-p_{\mathrm{a}}(\mathrm{SMR})\right]$ when available (Table 2).

Table 1 Differences $\left(d_{\mathrm{e}} \pm \mathrm{SE}\right)$ between the proportion of eggs layed on G medium by SMG and SMR females (parents sampled from $\mathrm{G}$ and from $\mathrm{R}$ medium, respectively)

\section{Base population}

\begin{tabular}{lrrrr} 
Treatment & $\mathrm{A}$ & $\mathrm{B}$ & $\mathrm{C}$ & $\mathrm{D}$ \\
\hline First evaluation & & & & \\
R $+\mathrm{G}$ & $0.002 \pm 0.142$ & $0.195 \pm 0.051^{*}$ & $0.214 \pm 0.062^{*}$ & $-0.056 \pm 0.071$ \\
R/G & $-0.045 \pm 0.044$ & $0.114 \pm 0.045^{*}$ & $-0.127 \pm 0.059 \dagger$ & $0.000 \pm 0.079$ \\
Second evaluation & & & & \\
R +G & & & $-0.101 \pm 0.050$ & $0.264 \pm 0.065^{*}$ \\
R/G & $0.389 \pm 0.056^{* *}$ & $0.181 \pm 0.035^{*}$ & $-0.290 \pm 0.050 \dagger \dagger$ & $-0.105 \pm 0.058$ \\
R & $0.029 \pm 0.055$ & $0.106 \pm 0.087$ & $0.025 \pm 0.068$ & $0.220 \pm 0.084$ \\
G & $0.070 \pm 0.082$ & $0.024 \pm 0.050$ & $0.250 \pm 0.033^{* *}$ & \\
\hline
\end{tabular}

${ }^{*} P<0.05 ;{ }^{*} P<0.01$ in the sequential Bonferroni test, performed for each table's row, checking whether the difference $\left(d_{\mathrm{e}}\right.$ or $\left.d_{\mathrm{a}}\right)$ is greater than zero.

$\dagger P<0.05 ; \dagger P<0.01$ at a two tailed $t$-test.

Table 2 Differences $\left(d_{\mathrm{a}} \pm \mathrm{SE}\right)$ between the proportion of offspring of SMG and SMR parents emerged from G medium (first evaluation)

Base population

\begin{tabular}{|c|c|c|c|c|}
\hline Treatment & A & B & $\mathrm{C}$ & D \\
\hline $\mathrm{R}+\mathrm{G}$ & $0.118 \pm 0.045^{*}$ & $0.222 \pm 0.064^{*}$ & $0.149 \pm 0.032^{* *}$ & $0.102 \pm 0.022^{*}$ \\
\hline $\mathrm{R} / \mathrm{G}$ & $-0.020 \pm 0.046$ & $-0.012 \pm 0.040$ & $-0.013 \pm 0.036$ & $0.112 \pm 0.085$ \\
\hline
\end{tabular}

${ }^{*} P<0.05 ;{ }^{*} P<0.01$ in the sequential Bonferroni test, performed for each table's row, checking whether the difference $\left(d_{\mathrm{e}}\right.$ or $\left.d_{\mathrm{a}}\right)$ is greater than zero.

$\dagger P<0.05 ; \dagger \uparrow P<0.01$ at a two tailed $t$-test. 
For each treatment $(R+G, R / G$ and the control, whether $R$ or $G$ ), the same hypothesis was tested in every population (A, B, C or D). To reduce the probability of type-I errors in a set of equivalent tests, the existence of between-habitat genetic differentiation was tested using sequential Bonferroni tests (Rice, 1989). Thus, a different sequential Bonferroni test was used for each treatment $(R+G, R / G$, and the control, including $\mathrm{R}$ and $\mathrm{G}$ considered together), each evaluation $(\alpha 1, \alpha 2)$ and each biological stage $\left(p_{\mathrm{e}}, p_{\mathrm{a}}\right)$.

Differentiation occurred in all four $R+G$ populations. In the first evaluation, populations $A(R+G)$ and $\mathrm{D}(\mathrm{R}+\mathrm{G})$ show a similar oviposition pattern for $\mathrm{SMG}$ and SMR females. Nevertheless, the proportion of adult offspring emerging in $G$ medium is greater for SMG than for SMR females. Therefore, the two progenies differ in within-habitat viability. This shows that the two subpopulations, each coming from a different habitat, are genetically different with respect to within-habitat adaptation, each being more adapted to its own habitat (as expected under soft selection). In populations $\mathrm{B}(\mathrm{R}+\mathrm{G})$ and $\mathrm{C}(\mathrm{R}+\mathrm{G})$, the average probability of oviposition on $G$ is greater for those genotypes sampled on $G\left(d_{\mathrm{e}}>0\right)$. In these two populations, the significant positive values of $d_{\mathrm{a}}$ are of the same order as those of $d_{\mathrm{e}}$ and may be explained as a consequence of those $d_{\mathrm{c}}$ values.

In the second evaluation, 20 months later, differentiation for oviposition preference has evolved in populations $A(R+G)$ and $D(R+G)$ but has vanished in population $C(R+G)$.

Among $R / G$ populations, only $B(R / G)$ showed positive genetic differentiation for oviposition preference in the first evaluation but it was not maintained in the second. None of these populations showed differentiation for adult emergence.

The behaviour of $\mathrm{C}(\mathrm{R} / \mathrm{G})$ population deserves further comment. At both evaluations, $d_{\mathrm{e}}$ was negative and significantly different from zero using two-tailed $t$-tests (this is an ad hoc test regarding the first evalua- tion, but not in the second). Irrespective of the mechanism responsible for this genetic differentiation, the question remains of how genotypes that confer preference to oviposit on a given medium, tend to be sampled from the opposite medium. $p_{\mathrm{e}}$ and $p_{\mathrm{a}}$ values (Table 3 ) show that in population $C$, eggs tend to be laid on $G$ but adults tend to emerge mainly from $\mathrm{R}$ (in fact we obtain $p_{\mathrm{e}} \neq 0.5$ and $p_{\mathrm{a}} \neq 0.5$, both significant at the 0.001 level). Therefore, there seems to be a general tendency for larvae to develop on the medium that they were not originally placed on. As global viability was high, these results cannot be explained by differential viability. Thus, 583 adults survived out of 663 eggs layed by SMG females, and 500 of these adults emerged on $\mathrm{R}$ tubes (instead of the 583/2 expected if mortality in $G$ was responsible for the low $p_{\text {a }}$ value). Similarly, 550 adults emerged out of 781 eggs layed by SMR females, and 491 emerged in $\mathrm{R}$ tubes. In this respect, the analysis of the difference $F=\left(p_{\mathrm{e}}-p_{\mathrm{a}}\right)$, computed at each tube, can be illuminating because this $F$ value can be considered to be a measure of the larval flux from $G$ to $R$ medium. Taking an average over all tubes, we found that $F_{\mathrm{SMR}}-F_{\mathrm{SMG}}=0.120 \pm$ 0.065 , which is significantly greater than zero $(P<0.05)$. This means that genotypes sampled from $\mathrm{R}$ show a relative preference for oviposition on $G$, but produce larvae with a greater preference for $\mathrm{R}$ (relative to that of genotypes sampled from $G$ ). Despite the positive value for $F_{\mathrm{R}}-F_{\mathrm{G}}$, no positive $d_{\mathrm{a}}$ value was observed. This was probably due to inefficient larval choice in the small tubes $\left(2.3 \mathrm{~cm}^{2} \phi\right.$ instead of $5 \mathrm{~cm}^{2} \phi$ for the cage bottles) where this evaluation was performed.

In the six surviving control populations (evaluated in the second measurement), only CG gives a (positive) significant differentiation, as revealed by the corresponding Bonferroni test. Thus, populations maintained under homogeneous conditions seems to preserve less genetic variability for oviposition-site preference.

Table 3 Mean proportion of eggs $\left(p_{\mathrm{e}} \pm \mathrm{SE}\right)$ layed on $\mathrm{G}$ medium and that $\left(p_{\mathrm{a}} \pm \mathrm{SE}\right)$ of offspring emerging from the same, for SMG and SMR individuals (first evaluation, $R / G$ populations)

\begin{tabular}{|c|c|c|c|c|}
\hline \multirow[b]{2}{*}{ Population } & \multicolumn{2}{|l|}{ SMG } & \multicolumn{2}{|l|}{ SMR } \\
\hline & $p_{\mathrm{e}}$ & $p_{\mathrm{a}}$ & $p_{\mathrm{e}}$ & $p_{\mathrm{a}}$ \\
\hline $\mathrm{A}(\mathrm{R} / \mathrm{G}) \dagger$ & $0.328 \pm 0.036^{* * *}$ & $0.458 \pm 0.029$ & $0.468 \pm 0.048$ & $0.479 \pm 0.035$ \\
\hline $\mathrm{B}(\mathrm{R} / \mathrm{G}) \dagger$ & $0.452 \pm 0.037$ & $0.357 \pm 0.025^{* * *}$ & $0.366 \pm 0.037^{* *}$ & $0.369 \pm 0.032^{* * *}$ \\
\hline $\mathrm{C}(\mathrm{R} / \mathrm{G})$ & $0.744 \pm 0.056^{* * *}$ & $0.116 \pm 0.026^{* * *}$ & $0.871 \pm 0.020^{* * *}$ & $0.129 \pm 0.025^{* * *}$ \\
\hline $\mathrm{D}(\mathrm{R} / \mathrm{G})$ & $0.418 \pm 0.059$ & $0.560 \pm 0.072$ & $0.417 \pm 0.050$ & $0.448 \pm 0.047$ \\
\hline
\end{tabular}

$\dagger p_{\mathrm{e}}$ computed over the tubes' subsample where adult emergence was scored.

${ }^{*} P<0.05 ;{ }^{* *} P<0.01 ;{ }^{* * *} P<0.001$ as a two tailed $t$ to test $p_{\mathrm{c}} \neq 0.5\left(\right.$ or $\left.p_{\mathrm{a}} \neq 0.5\right)$. 
Table 4 Heritability $\left(h^{2} \pm \mathrm{SE}\right)$ estimated at December, 1986 for all populations (1), and at February, 1989 for $D(R+G), D(R / G)$ and their respective 'new' controls (2)

\begin{tabular}{|c|c|c|c|c|}
\hline \multirow[b]{2}{*}{ Population } & \multicolumn{4}{|l|}{ Treatment } \\
\hline & $\mathrm{R}$ & G & $R+G$ & $\mathrm{R} / \mathrm{G}$ \\
\hline $\mathrm{A}(1)$ & $0.455 \pm 0.037$ & $0.424 \pm 0.045$ & $0.585 \pm 0.040$ & $0.482 \pm 0.038$ \\
\hline$B(1)$ & $0.517 \pm 0.037$ & & $0.427 \pm 0.043$ & $0.438 \pm 0.041$ \\
\hline$C(1)$ & $0.625 \pm 0.044$ & $0.476 \pm 0.045$ & $0.430 \pm 0.035^{*}$ & $0.402 \pm 0.048^{*}$ \\
\hline$D(1)$ & $0.440 \pm 0.041$ & & $0.695 \pm 0.049^{*}$ & $0.677 \pm 0.049 *$ \\
\hline$D(2)$ & $0.458 \pm 0.042$ & $0.521 \pm 0.045$ & $0.375 \pm 0.040 \dagger$ & \\
\hline $\mathrm{D}(2)$ & $0.527 \pm 0.046$ & $0.555 \pm 0.041$ & & $0.515 \pm 0.037$ \\
\hline
\end{tabular}

*Significantly $(P<0.05)$ different from the $h^{2}$ of the $\mathrm{R}$ control using a sequential Bonferroni test.

$\dagger$ Significantly $(P<0.05)$ different from the $h^{2}$ of the $\mathrm{G}$ control using a sequential Bonferroni test.

Table 4 shows all heritability estimates $\left(h^{2}\right)$ for SBN. If environmental heterogeneity was relevant to the maintenance of the genetic variability of the character, larger $h^{2}$ values are expected in populations maintained in heterogeneous environments (whether $R+G$ or $\mathrm{R} / \mathrm{G}$ ) than in controls. Otherwise, drift can produce both negative or positive differences. We do not expect, however, $h^{2}$ to be the same for all the experimental populations derived from the same base population. Therefore, a two-tailed $t$-test was used for each difference $\left(h^{2}\right.$ of a population maintained under heterogeneous conditions $-h^{2}$ of a control derived from the same base population). Thus, we have a large number of non-independent comparisons in order to check the hypothesis of higher heritability for $R+G$ and $R / G$ populations. We checked the significance of these differences using a single sequential Bonferroni test (Rice, 1989) for all the $h^{2}$ estimated in December, 1986. There were four significant differences, two of them negative. Moreover, one-half of the non-significant differences also were negative. Thus, the overall results agree with the expectations derived from drift. Nevertheless, the results obtained for D populations may indicate the existence of genetic variability maintained by the heterogeneous nature of the environment [the heritability being significantly larger in both $\mathrm{D}(\mathrm{R}+\mathrm{G})$ and $\mathrm{D}(\mathrm{R} / \mathrm{G})]$. As $\mathrm{DG}$ was accidentally lost, we derived new $R$ and $G$ controls from both $D(R+G)$ and $\mathrm{D}(\mathrm{R} / \mathrm{G})$. Heritability in these new controls and their parentals $[D(R+G)$ and $D(R / G)]$, estimated 15 months after the control's foundation, together with the results of the corresponding sequential Bonferroni test, are also presented in Table 4 . This additional data suggest that the high $h^{2}$ previously observed in $D(R+G)$ and $D(R / G)$ was not due to the heterogeneous nature of the corresponding environment.
Table 5 shows the components of variance $\left(\sigma^{2}\right)$ for the random factors, and the sums of the quadratic effects $\left(\theta^{2}\right)$ for the fixed factors, both estimated from the corresponding analysis of variance. The effect of the nursing medium (NM) was often significant, revealing a moderate effect of the habitat on the character's expression. The corresponding component of the environmental variance seems to show temporal variation, which can be attributed to inadvertent differences in the preparation of the media. The usually significant between-tubes variance may be due both to genetic (between-family genetic variance) and environmental differences between tubes. No consistent temporal increase in this component was observed, except, perhaps, in populations $\mathrm{C}(\mathrm{R} / \mathrm{G}), \mathrm{D}(\mathrm{R}+\mathrm{G})$ and $\mathrm{D}(\mathrm{R} / \mathrm{G})$. In the last two cases, this observation, together with the high $\mathrm{D}(\mathrm{R}+\mathrm{G})$ and $\mathrm{D}(\mathrm{R} / \mathrm{G})$ estimated heritabilities, indicates a possible increase in the genetic variance of the character in these populations, which does not seem to have been caused by the heterogeneous nature of the environment.

It is striking that the medium from which parents were sampled (SM) never showed a significant effect on the expression of the character (just random type-I error is expected to produce one or more significant results out from the 28 tests performed). The interaction effect was, in general, non-significant. Thus, no between-habitat genetic differentiation was observed with respect to sternopleural bristle number.

\section{Discussion}

About 2 years after the start of the experiment (first evaluation), the four $R+G$ populations showed genetic between-habitat differentiation for oviposition-site preference and/or within-habitat viability. Habitat 
Table $5 \sigma^{2}$ and $\theta^{2}$ values estimated from the analysis performed to detect betweenhabitat differentiation with respect to SBN

\begin{tabular}{|c|c|c|c|c|c|}
\hline \multirow[b]{2}{*}{ Population } & \multirow[b]{2}{*}{ SV } & \multicolumn{4}{|c|}{ Measurement } \\
\hline & & I & II & III & IV \\
\hline $\mathrm{A}(\mathrm{R}+\mathrm{G})$ & SM & 0.024 & -0.027 & 0.011 & 0.014 \\
\hline $\mathrm{A}(\mathrm{R}+\mathrm{G})$ & NM & $1.131^{* * *}$ & $0.698^{* * *}$ & 0.103* & 0.041 \\
\hline$A(R+G)$ & I & -0.053 & -0.044 & -0.002 & $0.247^{*}$ \\
\hline$A(R+G)$ & $\mathrm{T}$ & $1.874^{* * *}$ & $1.059^{* * * *}$ & 0.481 & $1.071^{* * *}$ \\
\hline $\mathrm{A}(\mathrm{R}+\mathrm{G})$ & $\mathrm{E}$ & 2.682 & 3.382 & 3.837 & 3.410 \\
\hline $\mathrm{A}(\mathrm{R} / \mathrm{G})$ & SM & -0.031 & -0.009 & 0.011 & -0.011 \\
\hline $\mathrm{A}(\mathrm{R} / \mathrm{G})$ & NM & 0.025 & $0.411^{* * *}$ & $0.158^{*}$ & $0.289^{* *}$ \\
\hline $\mathrm{A}(\mathrm{R} / \mathrm{G})$ & I & 0.175 & 0.009 & -0.066 & -0.045 \\
\hline $\mathrm{A}(\mathrm{R} / \mathrm{G})$ & $\mathrm{T}$ & $1.617^{* * * * * *}$ & $0.875^{*}$ & $1.410^{* * * *}$ & $1.337^{* * *}$ \\
\hline $\mathrm{A}(\mathrm{R} / \mathrm{G})$ & $\mathrm{E}$ & 3.732 & 4.465 & 3.940 & 3.510 \\
\hline $\mathrm{B}(\mathrm{R}+\mathrm{G})$ & SM & 0.018 & -0.039 & -0.006 & -0.018 \\
\hline $\mathrm{B}(\mathrm{R}+\mathrm{G})$ & NM & 0.015 & 0.020 & 0.096 & -0.025 \\
\hline $\mathrm{B}(\mathrm{R}+\mathrm{G})$ & I & 0.014 & 0.094 & -0.031 & -0.070 \\
\hline $\mathrm{B}(\mathrm{R}+\mathrm{G})$ & $\mathrm{T}$ & $1.139^{* * *}$ & $1.628^{* * *}$ & $1.735^{* * *}$ & $1.281^{* *}$ \\
\hline $\mathrm{B}(\mathrm{R}+\mathrm{G})$ & $\mathrm{E}$ & 3.630 & 4.547 & 3.807 & 4.467 \\
\hline $\mathrm{B}(\mathrm{R} / \mathrm{G})$ & SM & -0.008 & -0.012 & -0.031 & -0.026 \\
\hline $\mathrm{B}(\mathrm{R} / \mathrm{G})$ & NM & $0.083^{*}$ & $0.188^{*}$ & $0.196^{* *}$ & 0.057 \\
\hline $\mathrm{B}(\mathrm{R} / \mathrm{G})$ & I & -0.045 & 0.088 & -0.061 & -0.047 \\
\hline $\mathrm{B}(\mathrm{R} / \mathrm{G})$ & $\mathbf{T}$ & $1.350^{* * *}$ & $1.519^{* * *}$ & $1.395^{* * *}$ & 0.211 \\
\hline $\mathrm{B}(\mathrm{R} / \mathrm{G})$ & $\mathrm{E}$ & 2.850 & 4.190 & 3.552 & 4.970 \\
\hline$C(R+G)$ & SM & -0.021 & 0.013 & 0.010 & \\
\hline $\mathrm{C}(\mathrm{R}+\mathrm{G})$ & NM & 0.063 & $0.327^{* * *}$ & 0.016 & \\
\hline$C(R+G)$ & I & $0.213^{*}$ & -0.061 & -0.026 & \\
\hline$C(R+G)$ & $T$ & $1.400^{* * *}$ & $0.979^{*}$ & $1.403^{* * *}$ & \\
\hline $\mathrm{C}(\mathrm{R}+\mathrm{G})$ & $\mathrm{E}$ & 3.427 & 4.107 & 3.040 & \\
\hline $\mathrm{C}(\mathrm{R} / \mathrm{G})$ & SM & -0.021 & -0.023 & -0.023 & \\
\hline $\mathrm{C}(\mathrm{R} / \mathrm{G})$ & NM & 0.016 & $0.132 *$ & 0.013 & \\
\hline$C(R / G)$ & I & 0.026 & -0.017 & -0.044 & \\
\hline $\mathrm{C}(\mathrm{R} / \mathrm{G})$ & $\mathrm{T}$ & $0.463^{*}$ & $0.853^{*}$ & $0.812^{*}$ & \\
\hline $\mathrm{C}(\mathrm{R} / \mathrm{G})$ & $\mathrm{E}$ & 3.210 & 3.782 & 3.140 & \\
\hline $\mathrm{D}(\mathrm{R}+\mathrm{G})$ & SM & -0.018 & 0.009 & 0.066 & \\
\hline $\mathrm{D}(\mathrm{R}+\mathrm{G})$ & NM & $0.752^{* * * *}$ & $0.294^{* * *}$ & $0.176^{*}$ & \\
\hline $\mathrm{D}(\mathrm{R}+\mathrm{G})$ & I & 0.102 & 0.017 & 0.008 & \\
\hline $\mathrm{D}(\mathrm{R}+\mathrm{G})$ & $\mathrm{T}$ & $1.544^{* * *}$ & $2.045^{* * *}$ & $1.934^{* * *}$ & \\
\hline $\mathrm{D}(\mathrm{R}+\mathrm{G})$ & $\mathrm{E}$ & 3.937 & 3.478 & 3.195 & \\
\hline $\mathrm{D}(\mathrm{R} / \mathrm{G})$ & SM & -0.040 & 0.064 & -0.038 & \\
\hline $\mathrm{D}(\mathrm{R} / \mathrm{G})$ & NM & $0.716^{\text {*** }}$ & -0.042 & 0.019 & \\
\hline $\mathrm{D}(\mathrm{R} / \mathrm{G})$ & I & $0.369^{*}$ & -0.067 & -0.034 & \\
\hline $\mathrm{D}(\mathrm{R} / \mathrm{G})$ & $T$ & $1.887^{* * * *}$ & $2.308^{* * *}$ & $2.440^{* * *}$ & \\
\hline $\mathrm{D}(\mathrm{R} / \mathrm{G})$ & E & 4.225 & 3.820 & 4.557 & \\
\hline
\end{tabular}

${ }^{*} P<0.05 ;{ }^{* *} P<0.01 ;{ }^{* * *} P<0.001$ in the corresponding $F$-test.

Sources of variation (SV): SM (sampling medium); NM (nursing medium);

$\mathrm{I}$ (interaction); $\mathrm{T}$ (tubes); E (error).

choice polymorphisms which are unassociated with genetic variability for within-habitat adaptation are not ESS (García-Dorado, 1987) (although they may be protected). Therefore, the differentiation for oviposition-site preference found in populations $B(R+G)$ and $C(R+G)$, could have been taken as a suggestion of differentiation for within-habitat viability in those populations. This has not been detected. Nevertheless, because density was not controlled during the evaluation, the differences between $d_{\mathrm{a}}$ and $d_{\mathrm{e}}$ may underestimate the differences in viability: i.e. for high density conditions, it is not true that the more eggs laid the 
more adults emerge (in particular, under soft selection, the number of adults emerged should be constant). Thus, although $d_{\mathrm{a}}>d_{\mathrm{e}}$ would suggest the existence of between-habitat genetic differentiation for withinhabitat adaptation, small absolute $d_{\mathrm{a}}$ values are meaningless. Populations $\mathrm{A}(\mathrm{R}+\mathrm{G})$ and $\mathrm{D}(\mathrm{R}+\mathrm{G})$ show differentiation only for within-habitat adaptation. In the second evaluation (about 20 months later), three of the four populations showed between-habitat genetic differentiation with respect to oviposition site preferences. In populations $A(R+G)$ and $D(R+G)$, this differentiation seems to have developed after the first measurement was made. This is in agreement with the theory, which predicts that genetic differentiation for habitat preference can evolve as a consequence of differentiation for within-habitat adaptation under soft selection (García-Dorado, 1986, 1987).

With respect to $\mathrm{R} / \mathrm{G}$ populations, two showed between-habitat genetic differentiation for oviposition site preference in the first evaluation. As SMR and SMG samples were split at the larval phase, this differentiation with respect to oviposition-site preference reveals some kind of between-habitat genetic differentiation at the larval phase. This may be due to habitat fidelity, habitat preference, or differential within-habitat viability. Non-significant $d_{\mathrm{a}}$ values may be due to the particular experimental conditions in which this evaluation was carried out: small tubes in which larvae (which show high mobility) may be unable to choose a single medium. The small (non-significant) $d_{\mathrm{e}}$ values for $\mathrm{A}(\mathrm{R} / \mathrm{G})$ and $\mathrm{D}(\mathrm{R} / \mathrm{G})$ show that strong habitat preference (or habitat fidelity) has not evolved at the larval level in these populations. In this circumstance, because larval mobility is high, oviposition-site preference is neutral (except, perhaps, through viability at the eclosion phase), environmental heterogeneity is fine-grained, and soft selection is not possible. Nevertheless, the absence [for $A(R / G)$ and $D(R / G)$ populations] of genetic variation for oviposition site preference has not been proven, and it might have been maintained as neutral variation. Only one population maintained differentiation for oviposition-site preference at the second evaluation.

Only one of six control populations showed genetic variability for oviposition-site preference at the end of the experiment (differentiation for adult emergence was not measured).

These results demonstrate that genetic variability for oviposition site preference and/or within-habitat viability is present in both natural and laboratory populations. They also suggest that such variability can be neutral in homogeneous environments, but could be actively protected under soft selection $(R+G$ populations). Nevertheless, protection is not strong enough to prevent the loss of between-habitat genetic differentiation from the first to the second evaluation in population $C(R+G)$, which may have occurred by drift. Furthermore, results from $\mathrm{R} / \mathrm{G}$ populations show that the definition of fine or coarse-grained environments during the selective phase is dependent on the individual's choice behaviour. Thus, efficient larval choice may occasionally allow for the protection of genetic variability in environments where no pure soft selection occurs. Nevertheless, this mechanism seems to confer a weaker protection of the genetic variability.

The behaviour of population $\mathrm{C}(\mathrm{R} / \mathrm{G})$ illustrates the possible consequences of the arbitrary nature of the environmental heterogeneity imposed in laboratory experiments. Natural population $\mathrm{C}$ (captured from the neighbourhood of a cellar just before its use in this experiment) may have had variability for ovipositionsite preference, larval preference and within-habitat adaptation, but the first character may have been negatively correlated to one of the others (or to both). Thus, genetic variability for oviposition-site preference, although perhaps neutral in the $R / G$ laboratory environment, may be maintained if it is (negatively) correlated with protected variability for larval preference and adaptation. On the other hand, in the presence of an initial negative correlation between oviposition-site preference and within-habitat adaptation, a kind of 'inverse' habitat fidelity may have evolved during the experiment, so that larvae tend to move from the habitat where the egg was laid to the opposite one where its fitness is greater. Genetic variability for larval preference would then appear as a consequence of oviposition-site preference and inverse habitat fidelity. The highly significant differentiation for oviposition-site preference observed in population CG is necessarily positive because, in our control populations, the sampling method (measurement $\alpha 2$ ) did not allow for larval migration. Therefore, genotypes that prefer to oviposite on a given habitat are necessarily sampled from that habitat. Such differentiation is due to genetic variance for oviposition site preference, which has probably been maintained as neutral.

In any case, our results show that genetic variability for larval preference can also exist in natural [and laboratory, see $B(R / G)]$ populations. A similar result was reported by Taylor \& Condra (1983), who found genetic variability for larval preference positively associated with within-habitat fitness in $D$. pseudoobscura.

Genetic differentiation for habitat preference and/or within-habitat viability has been detected for a series of populations that experience environmental heterogeneity (mainly $\mathrm{R}+\mathrm{G}$ populations). If the correspond- 
ing genetic variability was associated (whether pleiotropically or by linkage) with variability for SBN, between-habitat genetic differentiation for SBN could have occurred, and genetic variance for SBN would have been maintained (via soft selection) in the heterogeneous $R+G$ environments. Nevertheless, betweenhabitat genetic differentiation for SBN has not been observed at any stage for any population. Therefore, if $\mathrm{R}+\mathrm{G}$ or $\mathrm{R} / \mathrm{G}$ populations had maintained more genetic variance for SBN than control populations, this could have been ascribed to heterozygous advantage in heterogeneous habitats, and R/G populations would have been expected to maintain even more genetic variability for SBN than $R+G$ cages. On the contrary, the $h^{2}$ of SBN did not appear to be higher in populations that undergo environmental heterogeneity (whether $\mathrm{R}+\mathrm{G}$ or $\mathrm{R} / \mathrm{G}$ ). This suggests that MacKay's results, with respect to the maintenance of genetic variability for SBN in heterogeneous environments for ethanol content, cannot be extrapolated to other populations. Our finding is in agreement with results obtained by López-Fanjul \& Ruano (1987), who did not detect any consistent trend in the behaviour of SBN in different natural populations during their first six generations of maintenance in laboratory conditions. MacKay's observations may have been due to random drift or to the particular genetic characteristics of her base population. It must be noted that, in our case, heritability differences between populations (whether positive or negative) were larger when the base populations were recently captured (C and D). Linkage between loci controlling SBN and other loci relevant for adaptation to laboratory conditions may be responsible for the erratic behaviour of SBN heritability. Nevertheless, drift cannot be ruled out. It must also be noted that our conclusion refers to the genetic variability of SBN measured in a standard medium. Nevertheless, genotype-habitat interaction for SBN has not been detected in other experiments using habitats with different ethanol content (MacKay, 1981).

Our general conclusion is that soft selection occurred under the relevant environmental heterogeneity. This resulted in between-habitat genetic differentiation for different fitness components, for which some genetic variability was therefore maintained. However, this effect cannot be extended to the whole genome. In particular, our laboratory-simulated environmental heterogeneity (including that for ethanol content) does not seem to protect the genetic variance for SBN. This is in agreement with the classical view that, at least in the standard homogeneous laboratory medium, SBN is not under significant selection.

\section{Acknowledgements}

We thank A. Gallego, C. López-Fanjul and M. A. Toro for helpful comments, and to an anonymous referee for careful notes which greatly improved the manuscript. This work was supported by a CICYT grant (No. PB86-0017).

\section{References}

ABDEL REHIM, A. H. 1983. Difference in habitat selection by different morphs of the land snail Arianta arbustorum. Biol. J. Linn Soc., 20, 185-193.

BARTON, N. H., JONES, J. S. AND MALLET, J. 1988. No barriers to speciation. Nature, 336, 13-14.

BIRD, S. R. AND SOMEONOFF, R. 1986. Selection for oviposition preference in Drosophila melanogaster. Gen. Res., 48, $151-160$.

CAVENER, D. 1979. Preference for ethanol in Drosophila melanogaster associated with the Alcohol Dehydrogenase polymorphism. Behav. Genet., 9, 359-365.

GARCIA-DORADO, A. 1986. The effect of niche preference on polymorphism protection in a heterogeneous environment. Evolution, 40, 936-945.

GARCIA-DORADO, A. 1987. Polymorphism from environmental heterogeneity: Some features of genetically induced niche preference. Theor. Pop. Biol., 32, 66-75.

GARCIA-DORADO, A. 1990. The effect of soft selection on the variability of a quantitative trait. Evolution, 44, 168-179.

GUITTENBERG, E. 1988. Sympatric speciation in snails: A largely neglected model. Evolution, 42, 826-828.

HALEY, C. S. AND BIRLEY, A. J. 1983. The genetical response to natural environments. II. Observations on replicate populations in spatially varied laboratory environments. Heredity, 51, 581-606.

HILL, w. G. 1970. Design of experiments to estimate heritability by regression of offspring on selected parents. Biometrics, 26, 566-571.

HOEKSTRA, R. F., BILJSMA, R. AND DOLMAN, J. 1985. Polymorphism from environmental heterogeneity: models are only robust if the heterozygote is close in fitness to the favoured homozygote in each environment. Gen. Res., 45, 299-314.

HOFFMAN, A. 1985. Effect of experience on oviposition and attraction in Drosophila: comparing apples and oranges. Am. Nat., 126, 41-51.

JAENIKE, J. AND GRIMALDI, D. 1983. Genetic variation for host preference within and among populations of Drosophila tripunctata. Evolution, 37, 1023-1033.

JAENIKE, J. 1987. Genetics of oviposition-site preference in $D$ tripunctata. Heredity, 59, 363-369.

JONES, J. S. 1980. Can Genes choose habitats? Nature, 286 , 757.

JONES, J. S. 1982. Genetic differences in individual behaviour associated with shell polymorphism in the snail Cepea nemoralis. Nature, 298, 749-750. 
JONES, J. S. AND PROBERT, R. F. 1980. Habitat selection maintains a deletereous allele in a heterogeneous environment. Nature, 287, 632-633.

LERNER, 1. M. 1954. Genetic Homeostasis. Oliver and Boyd, Edinburgh.

LEVENE, H. 1953. Genetic equilibrium when more than one ecological niche is available. Am. Nat., 87, 331-333.

LOPEZ-FANJUL, C. AND RUANO, R. G. 1987. Indirect natural selection for bristle number induced by 'domestication' in populations of Drosophila melanogaster. Genét. Ibér., 39, 379-388.

MCDONALD, J. F. AND AYALA, F. J. 1974. Genetic response to environmental heterogeneity. Nature, 250, 572-574.

MACKAY, T. 1981. Genetic variation in varying environments. Gen. Res., 37, 79-93.

MAYNARD SMITH, J. 1966. Sympatric speciation. Am. Nat., 100, $637-650$.

MAYNARD SMITH, J. AND HOEKSTRA, R. 1980. Polymorphism in varied environment: how robust the models are? Gen. Res., 35, 45-57.

MINAWA, A. AND BIRLEY, A. J. 1978. The genetical response to natural selection by varied environments. I. Short-term observation. Heredity, 40, 39-50.

POWEL, J. R. AND WISTRAND, H. 1978. The effects of heterogeneous environments and a competitor on genetic variation in Drosophila. Am. Nat., 112, 935-947.

PROUT, T. 1968. Sufficient conditions for multiple niche polymorphism. Am. Nat., 102, 493-496.

RAUSHER, M. D. 1984. The evolution of habitat preference in subdivided populations. Evolution, 83, 596-608.

RAUSHER, M. D. 1985. Variability for host preference in insect populations: Mechanistic and evolutionary models. $J$. Insect. Physiol., 31, 873-889.
RICE, W. R. 1985. Disruptive selection on habitat preference and the evolution of reproductive isolation: An exploratory experiment. Evolution, 39, 645-656.

RICE, W. R. 1989. Analyzing tables of statistical tests. Evolution, 43, 223-225.

RICE, W. R. AND SALT, G. W. 1988. Speciation via disruptive selection on habitat preference: Experimental evidence. Am. Nat., 131, 911-917.

ROBERTSON, A. 1967. The nature of quantitative genetic variation. In: Brink, R. A. (ed.), Heritage from Mendel, University of Wisconsin Press, Wisconsin, USA, pp. 265-280.

SINGER, M. C. AND THOMAS, C. D. 1988. Heritability of oviposition preference and its relationship to offspring performance within a single insect population. Evolution, 42, 977-985.

TAYLOR, C. E. AND CONDRA, C. 1983. Resource partitioning among genotypes of D. pseudoobscura. Evolution, 37, 135-149.

THOMPSON, J. N. 1988. Evolutionary genetics of oviposition preference in swalowtail butterflies. Evolution, 42, 1223-1234.

VERDONK, M. 1987. Adaptation to environmental heterogeneity in populations of D. melanogaster. Gen. Res., 49, $1-10$.

VIA, S. 1986. Genetic covariance between oviposition preference and larval performance in an insect herbivore. Evolution, 40, 778-785.

YAMAZAKI, T., KUSAKABE, S., TACHIDA, H., ICHINOSE, M., YOSHIMARU, H., MATSUO, Y. AND MUKAY, T. 1983. Re-examination of diversifying selection of polymorphic allozyme genes by using population cages in Drosophila melanogaster. Proc. Natl Acad. Sci., 80, 5789-5792. 